\title{
Perfil de desempenho em teste de triagem de processamento auditivo (SCAN) em crianças de sete e oito anos residentes em Cuiabá
}

\author{
Auditory processing screening test (SCAN) performance profile in \\ seven and eight year-old children living in Cuiabá
}

\author{
Priscila de Araújo Lucas Rodrigues ${ }^{1}$, Koichi Sameshima², Elena Zaidan ${ }^{3}$
}

\begin{abstract}
RESUMO
Objetivo: Caracterizar o perfil de desempenho de crianças normo-ouvintes de sete e oito anos no teste de triagem de processamento auditivo SCAN. Métodos: O trabalho é um estudo observacional descritivo. Foram selecionadas 109 crianças de sete anos e 106 crianças de oito anos da rede regular de ensino na cidade de Cuiabá (MT), utilizando os seguintes critérios de inclusão: portadoras de limiares de audibilidade de até $15 \mathrm{~dB}$ NA; ausência de indicativos de distúrbio de processamento auditivo; e ausência de alterações fonológicas. As crianças selecionadas foram avaliadas por meio de um teste de triagem de processamento auditivo SCAN. As pontuações obtidas por essas crianças foram comparadas com a literatura nacional. Resultados: As médias e os desvios-padrões encontrados nos sub-testes fala filtrada, fala no ruído e palavras competitivas na idade de sete anos foram, respectivamente, $24,4 \pm 5,1,33,4 \pm 3,4$ e $76,5 \pm 9,7$ pontos, e na idade de oito anos, respectivamente, $24,0 \pm 4,8 ; 34,0 \pm 3,0$ e 77,5 $\pm 10,8$ pontos. Conclusão: As pontuações deste estudo no teste de triagem SCAN não podem ser generalizadas como valores de normalidade para todas as crianças brasileiras devido à variabilidade regional. Sendo assim, reforça-se a necessidade de estudos com casuísticas maiores que descrevam o desempenho de crianças de várias faixas etárias e pertencentes a diversas regiões geográficas e condições sociais.
\end{abstract}

Descritores: Testes auditivos; Audição; Percepção auditiva; Criança; Questionários

\section{INTRODUÇÃO}

A avaliação do processamento auditivo (PA) é um procedimento de grande valia na clínica fonoaudiológica, pois, pela precoce detecção e caracterização de alteração na capacidade de manipulação da mensagem sonora, é possível nortear a conduta terapêutica fonoaudiológica para maximizar a eficiência da comunicação oral. Na fonoaudiologia, preconiza-se uma avaliação composta de testes comportamentais e/ou eletrofisiológicos, que devem ser interpretados de forma conjunta e integrada. O principal objetivo da avaliação do processamento auditivo central em crianças é verificar a integridade e o estado de neuromaturação da via auditiva ${ }^{(1)}$.

Trabalho realizado na Faculdade de Medicina da Universidade de São Paulo - USP - São Paulo (SP), Brasil.

(1) Pós-graduanda em Ciências pelo Programa de Pós-Graduação em Fisiopatologia Experimental da Faculdade de Medicina da Universidade de São Paulo - USP - São Paulo (SP), Brasil.

(2) Livre-Docente, Professor Associado da Faculdade de Medicina da Universidade de São Paulo - USP - São Paulo (SP), Brasil.

(3) Pós-graduanda em Distúrbios da Comunicação pela University of Massachusetts - UMass - Amherst (MA), Estados Unidos.

Endereço para correspondência: Priscila de Araújo Lucas Rodrigues. R. Barão de Melgaço, 50/203, bl 05, Porto, Cuiabá - MT, CEP 78025-300.

E-mail: prilucas@hotmail.com

Recebido em: 6/7/2007; Aceito em: 29/2/2008
O processamento auditivo pode ser definido como os mecanismos responsáveis pelos comportamentos de localização sonora, discriminação auditiva, reconhecimento auditivo, separação binaural, figura fundo, bem como de resolução, integração e ordenação temporal ${ }^{(2-3)}$. O processamento auditivo também pode ser definido como o conjunto de habilidades específicas das quais o indivíduo depende para interpretar o que ouve $\mathrm{e}^{(4)}$.

O distúrbio do processamento auditivo (DPA) é uma entidade clínica de difícil diagnóstico, pois pode estar associado a uma ampla gama de entidades neuropatológicas ${ }^{(5)}$. Os portadores de DPA geralmente têm dificuldade de comunicar-se em ambientes ruidosos e de compreender anedotas e piadas, atenção reduzida, dificuldade de entender o que lêem, dificuldade com a linguagem expressiva (regras da língua) e na produção de determinados sons da fala, principalmente o $/ \mathrm{r} / \mathrm{e}$ $\mathrm{o} / l /$, além de baixo desempenho escolar, enquanto apresentam nível de inteligência normal ${ }^{(5-6)}$.

Além de fatores hereditários, o DPA pode ser causado por otites recorrentes, mormente no período de maturação das vias auditivas, e perda auditiva periférica decorrente de privação sensorial, que estas alterações acarretam. A incidência de DPA também está relacionada ao baixo nível socioeconômico, conseqüente ao estado de má nutrição, e aos baixos níveis de estimulação auditiva e lingüística de crianças nesse grupo social $^{(7-10)}$. 
O DPA está correlacionado com distúrbios de aprendizagem e déficits de atenção, com ressalva de que essas são entidades clínicas distintas, porém com possibilidades de coexistência ${ }^{(1-16)}$. A prevalência de distúrbio de aprendizagem em crianças em idade escolar varia de 5-10\% na população americana. E a prevalência de déficit de atenção varia entre 3-20\% nas crianças americanas. Em relação ao DPA esse valor é de 2-3\% ${ }^{(5)}$. Faltam estudos nacionais sobre a prevalência do DPA em crianças brasileiras.

Diante desse cenário, faz-se necessária à detecção precoce do DPA em crianças em idade escolar com o objetivo de minimizar os efeitos nocivos da persistência do DPA e melhorar o desempenho escolar dessas crianças. Atualmente há no Brasil a necessidade de uma padronização e validação de testes de triagem para avaliação de PA, traduzidos ou criados para o português brasileiro para que profissionais da educação possam utilizar estes recursos para triar crianças com dificuldades escolares decorrente do DPA.

Diante dessa demanda, foi adaptado para o português brasileiro o teste de triagem de PA denominado Screening Test for Auditory Processing Disorders (SCAN) ${ }^{(17)}$. Para isso, selecionou palavras pelo material didático utilizado em séries primárias do primeiro grau. As palavras escolhidas foram dissílabas e paroxítonas, pois essas representavam as características morfológicas de maior ocorrência no português brasileiro. Foi realizado um balanceamento fonético das listas de palavras utilizadas em um laboratório, com posterior gravação em estúdio. O teste SCAN foi, então, adaptado ao português com posterior aplicação em 60 crianças da faixa etária de seis a onze anos, sendo 10 de cada idade, sem indícios de DPA, com audição periférica normal e inclusas em escola particular da rede regular de ensino de São Paulo-SP. Por fim, o teste foi aplicado em 11 crianças com diagnóstico de DPA.

$\mathrm{O}$ teste SCAN foi idealizado e padronizado por um pesquisador americano e é bastante utilizado em escolas norteamericanas pelos profissionais da educação ${ }^{(18)}$. O SCAN serve para triar crianças com possíveis transtornos do sistema nervoso auditivo central identificando aquelas com risco de DPA, bem como aquelas que possam se beneficiar das estratégias específicas de reabilitação $0^{(19-20)}$. O SCAN foi elaborado para uso específico em ambiente escolar, tendo em vista todas as peculiaridades desse ambiente, tais como a limitação de equipamentos avaliativos e a disponibilidade de tempo.

Os resultados dos procedimentos de triagem devem ser analisados com cautela, não devendo ser finalizado o diagnóstico apenas com base em um procedimento de varredura como o ocorrido nas triagens. Caso haja uma alteração na triagem de PA, deve-se encaminhar a criança para uma avaliação completa que utilize uma bateria de testes e não somente um teste isolado, para minimizar os resultados falsos positivos ${ }^{(20-22)}$.

Tendo em vista uma futura padronização do teste SCAN em crianças brasileiras, foi realizado o presente estudo com o objetivo de caracterizar o padrão de normalidade da pontuação no teste SCAN, obtida por crianças de sete e oito anos residentes em Cuiabá (MT), bem como comparar os resultados obtidos no presente estudo com um estudo nacional ${ }^{(17)}$, cuja casuística foi menor e obtida em região geográfica brasileira distinta.

\section{MÉTODOS}

Este protocolo de pesquisa foi aprovado pela Comissão de Ética para Análise de Projetos de Pesquisa (CAPPesq) do Hospital das Clínicas da Faculdade de Medicina da Universidade de São Paulo sob número 361/06. O presente trabalho é um estudo observacional descritivo, no qual características relevantes do grupo avaliado serão relatadas e discutidas.

Inicialmente, foi calculada a casuística adequada para que a pontuação encontrada para cada grupo etário pudesse caracterizar o desempenho da população em geral residente em Cuiabá (MT). Assim, foram contatadas cerca de 400 crianças entre a faixa etária de sete e oito anos, pertencentes à rede regular de ensino de Cuiabá (MT). No primeiro contato, os pais foram argüidos por meio de um questionário, contendo perguntas para averiguar se a criança era uma candidata a apresentar DPA. Todas as crianças autorizadas pelos pais a participarem da pesquisa foram submetidas a uma avaliação de habilidades fonológicas por meio do teste Perfil de Habilidades Fonológicas ${ }^{(22)}$, com a finalidade de detectar alguma alteração lingüística que pudesse comprometer a coleta de dados e a análise do exame de PA. A seguir, foi realizada uma triagem audiológica, em que as crianças foram avaliadas por meio da audiometria tonal liminar por via aérea, na faixa de freqüências de 500 a $4000 \mathrm{~Hz}$. O audiômetro utilizado foi o modelo GSI 17 da marca Grason-Stadler ${ }^{\circledR}$ e fones modelo TDH 39 da marca Telephonics®. Foi realizada calibração biológica, pela avaliação de dez crianças de sete anos e de dez crianças de oito anos, para estabelecer o zero de referência para avaliação audiométrica em ambiente não tratado acusticamente.

Após os procedimentos iniciais de triagem anteriormente citados, os grupos selecionados foram compostos de 109 crianças de sete anos e 106 de oito anos, totalizando 215 crianças com as seguintes características: limiar de audibilidade de até $15 \mathrm{~dB}$ NA, ausência de alterações fonológicas, ausência de história pregressa que sugerisse a presença de distúrbio de processamento auditivo, de ambos os sexos, todas destras e matriculadas em uma escola particular da rede regular de ensino de Cuiabá (MT).

Os critérios de inclusão foram escolhidos, tendo em vista a eliminação de possíveis alterações na audição periférica e central dos indivíduos participantes, a fim de caracterizar o padrão de normalidade de pontuação da população estudada. A exclusão de algumas crianças ocorreu, por não apresentarem uma ou mais características já citadas ou pela não autorização dos responsáveis.

As crianças selecionadas $(n=215)$ foram submetidas à avaliação do PA por meio do teste SCAN versão em português, gravado em CD. Para tal procedimento, utilizou-se um tocador de CD estereofônico portátil, modelo CD-105 da marca Lenoxx Sound®, e fone modelo HP 195 da marca Philips®.

O teste SCAN é um procedimento de fácil aplicação, podendo ser utilizado em crianças de três a 11 anos de idade. $\mathrm{O}$ teste é composto pelos seguintes três sub-testes:

a. Fala Filtrada: é um sub-teste de apresentação monoaural em que duas listas distintas de 20 palavras são apresentadas a cada uma das orelhas separadamente. Os sons das palavras deste sub-teste sofreram um tratamento acústico 
no qual as freqüências que compõem as palavras das listas foram modificadas por um filtro passa-baixa (até $1000 \mathrm{~Hz}$ ) e com uma rejeição de $18 \mathrm{~dB}$ por oitava. Quatro palavras iniciais são de treinamento.

b. Fala no Ruído: é um sub-teste de apresentação monótica de palavras em que aos sons das palavras adicionou-se digitalmente ruído de murmúrio de conversação (babble). Neste sub-teste também são apresentadas monoauralmente duas listas de 20 palavras, distintas das anteriores, e quatro palavras de treinamento no início do sub-teste. A relação entre o sinal de fala e o ruído é de $+5 \mathrm{~dB}$, ou seja, o sinal é $5 \mathrm{~dB}$ mais elevado que o ruído.

c. Palavras Competitivas: é um teste dicótico em que pares de palavras distintas apresentadas simultaneamente em ambas às orelhas precisam ser reconhecidas. Este sub-teste é composto de uma lista de 50 pares de palavras no qual cada par é apresentado simultaneamente, uma palavra em cada orelha. Quatro pares de palavras iniciais são utilizados para treinamento.

Todas as avaliações foram feitas na própria escola e tiveram aproximadamente 30 minutos de duração ao todo por criança. A sala utilizada para a aplicação dos exames era a mais silenciosa da escola. A intensidade utilizada para realização dos exames de PA era regulada pelo controle de volume do tocador de $\mathrm{CD}$, de acordo com o nível de intensidade mais confortável para cada criança.

Os resultados foram anotados em um formulário para futura análise estatística, que foi realizada por meio dos programas Microsoft Excel e MINITAB 14. Fez-se, inicialmente, uma análise descritiva, utilizando valores absolutos de acertos, determinando-se a média, a mediana, os valores mínimo e máximo e o desvio-padrão. A fim de realizar comparação entre os desempenhos obtidos por idade em cada sub-teste, e também, por cada orelha em cada sub-teste, utilizou-se o teste não paramétrico de Wilcoxon com nível de significância de 5\%. Para comparação dos desempenhos nos sub-testes entre os grupos de crianças testadas neste estudo com o estudo nacional $^{(17)}$, realizou-se o teste não paramétrico de Mann-Whitney, com nível de significância de 5\%. Verificou-se que as distribuições estatísticas dos dados se desviavam significativamente da distribuição gaussiana.
Ressalta-se que, cada palavra repetida com sucesso correspondeu a um ponto e que a pontuação máxima possível no sub-teste fala filtrada e fala no ruído é de 40 pontos para cada sub-teste; e de 100 pontos para o sub-teste palavras competitivas, totalizando, portanto, 180 pontos.

\section{RESULTADOS}

\section{Estatística descritiva do teste SCAN nas populações de sete e oito anos}

Para análise das distribuições de pontuação obtida em cada orelha e sub-teste, bem como, na pontuação total do teste SCAN, calculou-se a estatística descritiva dos dados, contendo média, mediana, valor mínimo e máximo, e desvio-padrão dos acertos absolutos de cada sub-teste. O sumário dos valores descritivos deste estudo encontra-se na Tabela 1.

\section{Análise estatística inferencial comparativa entre os gru- pos etários neste trabalho}

Para comparação dos desempenhos entre os grupos etários em cada sub-teste do SCAN, utilizou-se o teste estatístico não paramétrico de Wilcoxon para variáveis com distribuição não gaussiana (Tabela 2).

Os p-valores das comparações de desempenho pelo teste estatístico não paramétrico de Wilcoxon entre as duas faixas etárias nos sub-testes, fala filtrada, fala no ruído, palavras competitivas e pontuação total foram 0,48, 0,20, 0,28 e 0,46, respectivamente. Portanto, estas análises não mostraram diferenças estatisticamente significantes nos desempenhos entre as crianças das duas faixas etárias em nenhum dos três sub-testes do SCAN, ou da pontuação total.

\section{Análise estatística inferencial comparativa entre as orel- has testadas neste trabalho}

As comparações das pontuações de cada orelha em cada sub-teste do SCAN foram feitas por meio da estatística não paramétrica de Wilcoxon (Tabela 3).

Os p-valores do teste de Wilcoxon das comparações dos

Tabela 1. Análise descritiva das pontuações obtidas pelas crianças de sete e oito anos em cada sub-teste do SCAN

\begin{tabular}{|c|c|c|c|c|c|c|c|c|c|c|c|c|c|}
\hline & \multirow[b]{2}{*}{ Sub-testes } & \multicolumn{3}{|c|}{ Média $\pm \mathrm{dp}$} & \multicolumn{3}{|c|}{ Mediana } & \multicolumn{3}{|c|}{ Mínimo } & \multicolumn{3}{|c|}{ Máximo } \\
\hline & & OD & OE & TOT & OD & OE & TOT & OD & OE & TOT & OD & OE & TOT \\
\hline \multirow{4}{*}{ 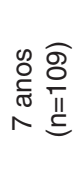 } & Fala Filtrada & $12,5 \pm 2,7$ & $12,0 \pm 3,0$ & $24,4 \pm 5,1$ & 12 & 12 & 25 & 5 & 5 & 13 & 18 & 18 & 34 \\
\hline & Fala no Ruído & $17,0 \pm 2,1$ & $16,4 \pm 2,0$ & $33,4 \pm 3,4$ & 17 & 17 & 34 & 9 & 9 & 22 & 20 & 20 & 39 \\
\hline & Palavras Competitivas & $40,1 \pm 4,9$ & $36,4 \pm 7,0$ & $76,5 \pm 9,7$ & 41 & 37 & 78 & 24 & 11 & 50 & 48 & 48 & 94 \\
\hline & Pontuação Total & & $134,3 \pm 14,6$ & & & 137 & & & 93 & & & 163 & \\
\hline \multirow{4}{*}{ 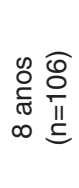 } & Fala Filtrada & $12,2 \pm 2,8$ & $11,8 \pm 2,8$ & $24,0 \pm 4,8$ & 12 & 12 & 24 & 5 & 5 & 12 & 20 & 17 & 35 \\
\hline & Fala no Ruído & $17,4 \pm 1,8$ & $16,7 \pm 1,9$ & $34,1 \pm 3,0$ & 18 & 17 & 34 & 13 & 11 & 27 & 20 & 20 & 39 \\
\hline & Palavras Competitivas & $40,0 \pm 5,9$ & $37,7 \pm 6,4$ & $77,5 \pm 10,8$ & 41 & 39 & 80 & 10 & 20 & 32 & 49 & 47 & 94 \\
\hline & Pontuação Total & & $135,5 \pm 14,6$ & & & 137 & & & 82 & & & 161 & \\
\hline
\end{tabular}

Legenda: $\mathrm{OD}$ = orelha direita; $\mathrm{OE}$ = orelha esquerda; $\mathrm{TOT}$ = total; $\mathrm{dp}=$ desvio-padrão 
Tabela 2. P-valores encontrados após a aplicação do teste Wilcoxon para comparação entre as pontuações obtidas no atual estudo entre as faixas etárias

\begin{tabular}{lcccc}
\hline Sub-testes SCAN & Fala Filtrada & Fala no Ruído & Palavras Competitivas & Total \\
\hline Mediana (7 anos) & 25 & 34 & 78 & 137 \\
Mediana (8 anos) & 24 & 34 & 80 & 137 \\
P-valor & 0,48 & 0,20 & 0,28 & 0,46 \\
\hline
\end{tabular}

desempenhos entre as orelhas nos sub-testes fala filtrada, fala no ruído e palavras competitivas foram para a população de sete anos, respectivamente, 0,30,0,012 e 0,01. Por sua vez para a população de oito anos esses valores foram 0,68, 0,0045 e 0,007 , respectivamente. Ou seja, observou-se diferenças estatisticamente significantes entre as orelhas nos sub-testes fala no ruído e palavras competitivas, em ambos os grupos etários. No sub-teste fala filtrada não se observou diferença de desempenho estatisticamente significativa entre as orelhas.

Tabela 3. P-valores encontrados após a aplicação do teste Wilcoxon para comparação entre as pontuações obtidas pelas orelhas nos subtestes do SCAN no atual estudo

\begin{tabular}{lccc}
\hline Sub-testes SCAN & $\begin{array}{c}\text { Fala } \\
\text { Filtrada }\end{array}$ & $\begin{array}{c}\text { Fala no } \\
\text { Ruído }\end{array}$ & $\begin{array}{c}\text { Palavras } \\
\text { Competitivas }\end{array}$ \\
\hline Mediana OD (7 anos) & 12 & 17 & 41 \\
Mediana OE (7 anos) & 12 & 17 & 37 \\
P-valor (7 anos) & 0,30 & 0,012 & 0,01 \\
\hline Mediana OD (8 anos) & 12 & 18 & 41 \\
Mediana OE (8 anos) & 12 & 17 & 39 \\
P-valor (8 anos) & 0,68 & 0,0045 & 0,007 \\
\hline
\end{tabular}

Legenda: $\mathrm{OD}=$ orelha direita; $\mathrm{OE}=$ orelha esquerda

\section{Análise estatística inferencial comparativa entre este trabalho e um estudo nacional}

Analisando o desempenho das populações estudadas neste trabalho e no estudo nacional avaliado ${ }^{(17)}$ em ambos grupos etários, sete e oito anos, observou-se diferença estatisticamente significante entre os desempenhos das orelhas direita, esquerda e na pontuação final do sub-teste fala filtrada, quando comparados os grupos de ambos os estudos.

No sub-teste fala no ruído, não houve uma diferença estatisticamente significante entre os desempenhos das orelhas direita, esquerda, bem como, na pontuação final do sub-teste em ambos os grupos etários.

Finalmente, no sub-teste palavras competitivas foi observado uma diferença estatisticamente significante entre pontuações das orelhas direita, esquerda e total do sub-teste em ambas as faixas etárias.

Analisando a pontuação total do teste SCAN, encontrou-se uma diferença estatisticamente significante entre os dois grupos comparados, a saber, crianças de sete anos deste trabalho e do estudo nacional ${ }^{(17)}$ e também, crianças de oito anos de ambos os trabalhos. Estes resultados estão sumariados na Tabela 4.

\section{DISCUSSÃO}

Em um estudo ${ }^{(24)}$ cujo objetivo foi determinar a presença de possíveis vieses socioculturais na análise do teste SCAN, os autores aplicaram-no em crianças pertencentes a dois grupos étnicos nos Estados Unidos da América: anglo-americano e latino-americano. Nesse estudo, os autores verificaram que não houve diferenças significativas entre as pontuações obtidas, quando comparados os grupos entre si, porém, ocorreram pontuações diversas dentro do mesmo grupo étnico, sugerindo, portanto, cautela ao analisar os resultados individuais desse teste. Essas diferenças encontradas em um mesmo grupo étnico, também puderam ser constatadas no presente estudo, em que houve uma grande diferença entre os valores mínimo e máximo obtidos pelas crianças testadas. É recomendado que nos testes de triagem, os resultados individuais obtidos por grupos de etnia e de nível social diverso sejam interpretados

Tabela 4. P-valores do teste Mann-Whitney para comparações de pontuações obtidas no atual estudo e no de Zaidan (2001) ${ }^{(17)}$

\begin{tabular}{|c|c|c|c|c|c|c|c|c|c|c|}
\hline \multirow{2}{*}{ Trabalhos } & \multicolumn{3}{|c|}{ Fala Filtrada } & \multicolumn{3}{|c|}{ Fala no Ruído } & \multicolumn{3}{|c|}{ Pal. Competitivas } & \multirow{2}{*}{$\begin{array}{c}\text { Pont. Total } \\
\text { SCAN }\end{array}$} \\
\hline & OD & OE & TOTAL & OD & OE & TOTAL & OD & OE & TOTAL & \\
\hline Zaidan $^{(17)}$ & 16 & 16 & 32 & 18 & 16 & 34 & 44 & 42 & 86 & 153 \\
\hline $\begin{array}{l}\text { Atual } \\
\text { trabalho }\end{array}$ & 12 & 12 & 25 & 17 & 17 & 34 & 41 & 37 & 78 & 137 \\
\hline 7 anos & $<0,0001^{*}$ & $<0,0001^{*}$ & $<0,0001^{*}$ & 0,11 & 0,93 & 0,44 & $0,005^{*}$ & $0,009^{*}$ & $0,0013^{*}$ & $<0,0001^{*}$ \\
\hline Zaidan $^{(17)}$ & 17 & 17 & 36 & 17 & 18 & 35 & 46 & 42 & 87 & 156 \\
\hline $\begin{array}{l}\text { Atual } \\
\text { trabalho }\end{array}$ & 12 & 12 & 24 & 18 & 17 & 34 & 41 & 39 & 80 & 137 \\
\hline 8 anos & $<0,0001^{*}$ & $<0,0001^{*}$ & $<0,0001^{*}$ & 0,94 & 0,18 & 0,44 & $<0,0001^{*}$ & $0,04^{*}$ & $0,0007^{*}$ & $<0,0001^{*}$ \\
\hline
\end{tabular}

Legenda: * valores estatisticamente significantes; $\mathrm{OD}=$ orelha direita; $\mathrm{OE}=$ orelha esquerda; $\mathrm{Pal}=\mathrm{Palavras;}$ Pont = Pontuação 
com cautela ${ }^{(18)}$. Dessa forma, evita-se a exposição de crianças a avaliações desnecessárias, bem como, poupa-se os pais da ansiedade gerada por uma falsa suspeita de alteração, além de evitar a inclusão de crianças auditivamente normais, no grupo de indivíduos inapropriadamente indicados como possíveis portadores de DPA ${ }^{(23)}$.

Foi realizado um estudo com o objetivo de verificar a maturação das habilidades de processamento auditivo central em crianças com e sem dificuldades escolares, nas faixas etárias de oito, nove e dez anos ${ }^{(1)}$. As autoras concluíram que as habilidades de processamento auditivo se aprimoraram ao longo dos anos em ambos os grupos avaliados, porém as crianças com dificuldades escolares apresentaram um desempenho inferior ao das crianças sem dificuldades. Além disso, a melhora no desempenho observada no grupo com dificuldade escolar foi mais abrupta entre as faixas etárias, sendo que no grupo sem dificuldades escolares o aprimoramento ocorreu de forma gradual entre as idades. Nesse estudo, as autoras fizeram uma análise entre as médias obtidas, de duas em duas faixas etárias, para verificar em qual a diferença estaria ocorrendo. Foi observado que as crianças de nove anos tinham um desempenho oscilando entre o padrão das crianças de oito e dez anos, podendo ser essa idade considerada como um período de transição.

Como não foi observada, neste estudo, uma diferença significativa entre as idades avaliadas, pode-se hipotetizar que a idade de oito anos poderia ser uma faixa etária de transição entre as crianças de sete e nove anos. Por outro lado, como a maturação do processamento auditivo ocorre de forma gradual em crianças sem DPA, não foi possível detectar uma diferença significante de desempenho entre as idades.

Os resultados do presente estudo mostraram maior pontuação da orelha direita sobre a esquerda nos sub-testes fala no ruído e palavras competitivas. Este achado corrobora com um estudo $^{(20)}$, cujo objetivo foi comparar os resultados de múltiplas combinações de testes de PA para averiguar qual era a melhor bateria de testes para identificar o DPA. Os autores observaram que, na aplicação do teste SCAN, houve um melhor desempenho da orelha direita sobre a esquerda. Esse achado pode ser explicado pela dominância do hemisfério esquerdo para as funções de linguagem em indivíduos destros.

No teste SCAN, a vantagem observada da orelha direita não pode ser atribuída ao fator aprendizagem, pois as listas de palavras apresentadas em cada uma das orelhas contêm palavras distintas. Além disso, essa vantagem não pode ser devido a ordem de apresentaçao dos estímulos, pois apesar de nos testes monóticos a orelha direita ser a primeira a ser avaliada, no teste palavras competitivas onde a apresentação das palavras é simultanea pode-se, ainda, perceber o melhor desempenho da orelha direita.

Os critérios de normalidade e validade do SCAN ainda não foram estabelecidos para a população brasileira. Para tanto, é necessária a realização de estudos multicêntricos que considerem as diferenças sociais, culturais e étnicas dos indivíduos testados. Para contribuir com esse processo, o presente estudo demonstrou o padrão de normalidade da pontuação do
SCAN aplicado em crianças normo-ouvintes de sete e oito anos residentes em Cuiabá (MT).

Ao comparar os resultados da pontuação total obtida pelos grupos de sete e oito anos, testados neste estudo com o trabalho nacional analisado ${ }^{(17)}$, percebe-se que houve uma diferença estatisticamente significante em ambas as idades testadas, confirmando a heterogeneidade do perfil de desempenho. Devido a possível variação de desempenho entre indivíduos com características diversas, ressalta-se que é mais prudente a consideração da pontuação total e não dos sub-testes de forma isolada $^{(18)}$.

Ressalta-se, contudo, que a pontuação total no teste SCAN é influenciada pelo desempenho em cada sub-teste, o que pode mascarar uma real dificuldade de processamento auditivo, gerando um resultado de triagem falso-negativo. Sendo assim, a avaliação por testes de processamento auditivo, deve considerar o desempenho nos mesmos, aliado às queixas auditivas percebidas no cotidiano.

Alguns autores concluíram que os dados normativos do teste SCAN para população americana não poderiam ser utilizados para avaliação de crianças residentes no Reino Unido, mostrando, mais uma vez, a necessidade de estudos multicêntricos ${ }^{(24)}$.

A motivação e a atenção são fatores que influenciam fortemente o desempenho de crianças em testes de $\mathrm{PA}^{(25)}$. No presente estudo, foi necessário algumas vezes interromper o processo de avaliação devido a dificuldades de concentração advindas da fadiga durante a aplicação do SCAN, pois o desempenho é dependente dos níveis de motivação e da atenção.

Estudos confirmam que o desempenho dos indivíduos testados pelo SCAN difere significativamente, dependendo do ambiente em que o mesmo é aplicado ${ }^{(26)}$. Ressalta-se, portanto, que os valores médios de pontuação discutidos no presente estudo, bem como, no trabalho nacional analisado ${ }^{(17)}$ foram obtidos pela aplicação do teste SCAN em ambiente escolar, para o qual foi elaborado.

\section{CONCLUSÕES}

- O perfil de pontuações obtidas neste estudo contribui como uma caracterização da pontuação de crianças normoouvintes residentes em Cuiabá - MT.

- As comparações dos resultados da aplicação do teste SCAN em crianças residentes em Cuiabá com outras residentes em São Paulo mostraram diferenças estatisticamente significantes entre sub-testes fala filtrada e palavras competitivas, sendo indicativos de significativas diferenças regionais mostrando que os valores encontrados em cada estudo não poderiam ser generalizados como valores de normalidade para as crianças todas as regiões brasileiras.

- Sendo assim, reforça-se a necessidade de estudos com casuísticas representativas que descrevam o desempenho de crianças de várias faixas etárias, regiões geográficas e condições socioeconômicas consideradas. 


\begin{abstract}
Purpose: To characterize the performance profile of seven- and eight-year-old children with normal hearing on the SCAN auditory processing screening test. Methods: This research is a descriptive observational study. One hundred and nine seven-year-old children and 106 eight-year-old children were recruited from the regular school system of Cuiabá (MT, Brazil) through the following criteria: auditory thresholds up to $15 \mathrm{~dB}$ NA; absence of auditory processing deficits; and absence of phonological disorders. Selected children were evaluated through SCAN auditory processing screening test. These children's scores were compared to two previous Brazilian studies. Results: Performance averages and standard-deviations for the sub-tests Filtered Words, Auditory Figure Ground and Competitive Words for seven-year-old children were, respectively, 24.4 $\pm 5.1,33.4 \pm 3.4$, and 76.5 \pm 9.7 points, and for eight-year-old children were, respectively, $24.0 \pm 4.8,34.0 \pm 3.0$, and $77.5 \pm 10.8$ points. Conclusion: SCAN test scores obtained for children in the present study cannot be generalized as normality values for Brazilian children, due to regional variability of children's performance in the test. It is emphasized the need for larger multi-centric studies, with greater number os subjects and encompassing children from many geographic regions and social conditions, as well as age ranges.
\end{abstract}

Keywords: Auditory tests; Hearing; Auditory perception; Child; Questionnaires

\title{
REFERÊNCIAS
}

1. Neves IF, Schochat E. Maturação do processamento auditivo em crianças com e sem dificuldades escolares. Pró-Fono. 2005;17(3):311-20.

2. ASHA: American Speech-Language Hearing Association. Central auditory processing: current status of research and implications for clinical practice. [cited $200514 \mathrm{Jul}$ ] Available from: http://chhs.sdsu. edu/slhs/publications/robin550.pdf; 1996.

3. ASHA: American Speech-Language Hearing Association. Central Auditory processing disorders: the role of the audiologist. Position statement. [cited $200614 \mathrm{Jul}$ ] Available from: www.asha.org/members/ deskref-journals/deskref/default; 2005.

4. Sanchez ML, Alvarez AMM, Cabete CF, Frazza MM. Avaliação do processamento auditivo em adultos. Acta AWHO. 2002;21(1).

5. Chermak GD, Musiek FE. Central auditory processing disorders: new perspectives. San Diego: Singular Publishing Group; c1997.

6. Schochat E. Processamento auditivo: atualidades em fonoaudiologia. São Paulo: Lovise; 1996.

7. Gravel JS, Wallace IF. Early otitis media, auditory abilities, and educational risk. Am J Speech Lang Pathol. 1995;4:89-94.

8. Carvallo RMM. Processamento auditivo: avaliação audiológica básica. In: Pereira LD, Schochat E. Processamento auditivo central: manual de avaliação. São Paulo: Lovise; 1997. p. 27-35

9. Mody M, Schwartz RG, Gravel JS, Ruben RJ. Speech perception and verbal memory in children with and without histories of otitis media. J. Speech Lang Hear Res. 1999;42(5):1069-79.

10. Santos MFC, Ziliotto KN, Monteiro VG, Hirata CHW, Pereira LD, Weckx LLM. Avaliação do processamento auditivo central em crianças com e sem antecedentes de otite média. Rev Bras Otorrinolaringol. 2001;67(4 Pt 1):448-54.

11. Rosen S. Auditory processing in dyslexia and specific language impairment: is there a deficit? What is its nature? Does it explain anything? J Phon. 2003;31(3-4):509-27.

12. Breier JI, Fletcher JM, Foorman BR, Klaas P, Gray LC. Auditory temporal processing in children with specific reading disability with and without attention deficit/hyperactivity disorder. J Speech Lang Hear Res. 2003;46(1):31-42.

13. Ferreira MIDC, Mello AM. Comorbidade entre transtorno de déficit de atenção e hiperatividade e distúrbio do processamento auditivo. Rev Fonoaudiol Brasil. 2006;4(2):1-3.
14. Rohde LA, Halpern R. Transtorno de déficit de atenção/hiperatividade: atualização. J Pediatr (Rio J). 2004;80(2 Suppl):S61-S70.

15. Elliott LL, Hammer MA. Longitudinal changes in auditory discrimination in normal children and children with language-learning problems. J Speech Hear Disord. 1988;53(4):467-74.

16. Kraus N, Koch DB, McGee TJ, Nicol TG, Cunningham J. Speechsound discrimination in school-age children: psychophysical and neurophysiologic measures. J Speech Lang Hear Res. 1999;42(5):1042-60.

17. Zaidan E. Desenvolvimento de uma bateria de testes de triagem da função auditiva central em pré-escolares e escolares na faixa etária de 6 a 11 anos. [dissertação mestrado]. São Paulo: Instituto de Psicologia da Universidade de São Paulo; 2001.

18. Keith RW. SCAN: A Screening Test for Central Auditory Processing Disorders. San Antonio: The Psychological Corporation; 1986.

19. Amos NE, Humes LE. SCAN test-retest reliability for first- and thirdgrade children. J Speech Lang Hear Res. 1998;41(4):834-45.

20. Domitz DM, Schow RL. A new CAPD battery - multiple auditory processing assessment: factor analysis and comparisons with SCAN. Am J Audiol. 2000;9(2):101-11. Comment in: Am J Audiol. 2002;11(1):7-9; author reply 9-12.

21. Singer J, Raymond MH, Preece JP. Effectiveness of central auditory processing tests with children. Am J Audiol. 1998;7:73-84.

22. Masquelier MP. Management of auditory processing disorders. Acta Otorhinolaryngol Belg. 2003;57(4):301-10.

23. Woods AG, Peña ED, Martin FN. Exploring possible sociocultural bias on the SCAN-C. Am J Audiol. 2004;13(2):173-84.

24. Marriage J, King J, Briggs J, Lutman ME. The reliability of the SCAN test: results from a primary school population in the UK. Br J Audiol. 2001;35(3):199-208.

25. Wightman F, Allen P, Dolan T, Kistler D, Jamieson D. Temporal resolution in children. Child Dev. 1989;60(3):611-24.

26. Emerson MF, Crandall KK, Seikel JA, Chermak GD. Observations on the use of SCAN to identify children at risk for central auditory processing disorder. Lang Speech Hear Serv Sch. 1997;28:43-9. 